\title{
A NOTE ON $f$-REGULARITY IN RINGS
}

\section{R. L. BLAIR}

1. Introduction. An element $a$ of a ring $A$ is regular, in the sense of von Neumann [6], ${ }^{1}$ in case $a=a x a$ for some element $x \in A$. Among the many variants of this notion is the notion of $f$-regularity [1]: An element $a \in A$ is said to be $f$-regular in case $a \in(a)^{2}$, where $(a)$ denotes the principal (two-sided) ideal of $A$ generated by $a$. (This is equivalent to the requirement that $(a)=(a)^{n}$ for every positive integer $n$.) A ring (ideal of a ring) is $f$-regular in case each of its elements is $f$ regular. We shall denote by $F(A)$ the set of all elements $a \in A$ such that $(a)$ is $f$-regular. It was observed in [1] that $F(A)$ is an $f$-regular ideal and indeed the largest $f$-regular ideal of $A$. This fact follows from the general considerations of $[3]$, as does the fundamental property $F(A / F(A))=0$. In $\S 2$ of this note we round out the list of "radicallike" properties of $F(A)$ by showing that if $B$ is an ideal of $A$ then $F(B)=B \cap F(A)$ and that, if $A_{n}$ denotes the complete matrix ring of order $n$ over $A$, then $F\left(A_{n}\right)=(F(A))_{n}$.

Introduction of the notion of $f$-regularity was, at least in part, the result of an attempt to find as large an ideal as possible with what may conveniently be described as "regularity" properties. In this connection we mention the following facts: (1) $F(A)$ surely contains all the ideals with regularity properties that are considered in [3]; thus for example $F(A)$ contains the largest weakly regular ideal of $A$. (2) $F(A)$ is large enough, so to speak, to induce a subdirect decomposition of $A$ (into what we have called $f$-primitive rings) when $F(A)=0$ (see $[1$, p. 147]). But just how large $F(A)$ actually is, however, is an open question. More precisely, it is not known whether $F(A)$ and the Jacobson radical $J(A)$ must have a nonzero intersection or (equivalently) whether an $f$-regular ring must be semi-simple. In fact, Theorem 3 below shows that the following statements are equivalent: (i) $J(A) \cap F(A)=0$ for every ring $A$; (ii) Every $f$-regular ring is semi-simple; (iii) Every simple ring is semi-simple. ${ }^{2}$ As is well known, the truth of (iii) is still in doubt. More precise information can be obtained, however, concerning the relationship between $F(A)$ and the prime radical $N(A)$ of McCoy [5]. For example, Theorem 4

Presented to the Society, June 19, 1954 ; received by the editors November 2, 1954.

${ }^{1}$ Numbers in brackets refer to the bibliography at the end of the paper.

2 By a "simple ring" we mean a ring which is not a zero ring and whose only ideals are itself and the zero ideal. The terms "semi-simple" and "radical ring" will be used in the sense of Jacobson. 
below asserts (among other things) that $N(A) \cap F(A)=0$ for every ring $A$.

We conclude this note by considering the effect on $F(A)$ of the descending chain condition for right ideals. Our final result (Theorem 5) states that in the presence of this chain condition $F(A)$ coincides with the largest regular ideal [2] of $A$.

2. Some fundamental properties of $F(A)$. In this section we obtain two important properties of the largest $f$-regular ideal $F(A)$ of $A$. It will be useful to observe first that $(a)^{3}=(a) a(a)$ for every $a \in A$. This follows directly from the fact that every element of $(a)$ is of the form $n a+u a+a v+\sum u_{i} a v_{i}$, where $n$ is an integer and the elements $u, v, u_{i}$, and $v_{i}$ are in $A$. If $B$ is an ideal of $A$ and $a \in B$, then $a$ generates an ideal of $B$ which we denote by $(a)_{B}$. We then have

Lemma 1. If $B$ is an ideal of the ring $A$ and if $a \in B$, then $(a)^{3} \subseteq(a)_{B}$.

Proof. We have $(a) \subseteq B$ so that $(a) a(a) \subseteq(a)_{B}$. Then $(a)^{3} \subseteq(a)_{B}$ by the above remark.

Now let $B$ be an ideal of $A$ and let $a \in F(B)$. Then $a \in(a)_{B}^{2} \subseteq(a)^{2}$ so that, using Lemma $1,(a)=(a)^{3} \subseteq(a)_{B}$. Hence $(a)=(a)_{B}$. We conclude that $(a)$ is an $f$-regular ideal of $A$ and therefore $a \in B \cap F(A)$. Conversely, let $a \in B \cap F(A)$ so that $a$ is an element of $B$ which is $f$-regular in $A$. But then, as one can easily see, $a$ is also $f$-regular in $B$. Thus $B \cap F(A)$ is an $f$-regular ideal of $B$ and hence $B \cap F(A) \subseteq F(B)$. We have proved

\section{THEOREM 1. If $B$ is an ideal of the ring $A$, then $F(B)=B \cap F(A)$.}

Theorem 1 shows, incidentally, that an $f$-regular ideal of $A$ is itself an $f$-regular ring.

We show next ${ }^{3}$ that if $A_{n}$ is the complete matrix ring of order $n$ over $A$, then $F\left(A_{n}\right)=(F(A))_{n}$. We begin by proving three lemmas. The first of these provides us with a convenient characterization of $f$-regularity:

Lemma 2. An element $a$ in $A$ is f-regular if and only if there exist elements $u_{i}, v_{i}$, and $w_{i}$ in $A$ such that $a=\sum u_{i} a v_{i} a w_{i}$.

Proof. If $a$ is $f$-regular, then $a \in(a)^{3}=(a) a(a)$ so that we have $a=\sum r_{j} a s_{j}$ with each $r_{j}$ and $s_{j}$ in $(a)=(a)^{3}$. But then each $r_{j}$ may be written as a sum of this same form. We thus obtain $a=\sum u_{i} a v_{i} a w_{i}$ for suitable elements $u_{i}, v_{i}$, and $w_{i}$ in $A$. The converse is clear.

${ }^{8}$ Our proof here is patterned after the proof of the corresponding result (Theorem 4) of [2]. 
Lemma 3. If $b$ is an element of $(a)^{2}$ such that $a-b$ is $f$-regular, then $a$ is f-regular.

Proof. We have $a-b \in(a-b)^{2} \subseteq(a)^{2}$ and therefore $a \in(a)^{2}$.

Our key lemma is the following

Lemma 4. If $A$ is an f-regular ring, then $A_{n}$ is an f-regular ring.

Proof. We consider first the case in which $n=2$. Let $\alpha$ be an element of $A_{2}$. By Lemma 2 we may express each element $a_{i j}$ of $\alpha$ in the form

$$
a_{i j}=\sum_{i}^{18} u_{i j}^{18} a_{i j} u_{i j}^{28} a_{i j} u_{i j}^{38}
$$

where the elements $u_{i j}^{r s}$ are in $A$. We may assume, moreover, that the range of $s$ is the same in each sum. Now denote by $[x]^{i j}$ the matrix in $A_{2}$ which has $x$ in the $(i, j)$ position and zeros elsewhere. Set

$$
\beta=\alpha-\sum_{s} \alpha_{s}
$$

where

$$
\alpha_{s}=\left[u_{12}^{18}\right]^{11} \alpha\left[u_{12}^{2 s}\right]^{21} \alpha\left[u_{12}^{3 s}\right]^{22},
$$

and note that $\alpha_{s}$ is in the ideal $(\alpha)^{2}$ of $A_{2}$. If for each $s$ we set

$$
\gamma_{r s}=\left[u_{11}^{r s}\right]^{11}+\left[u_{22}^{r s}\right]^{22} \quad(r=1,2,3)
$$

then a straightforward calculation shows that

$$
\delta=\beta-\sum_{s} \gamma_{18} \beta \gamma_{28} \beta \gamma_{38}=[x]^{21}
$$

for a suitable element $x \in A$. Again by Lemma 2 we may write $x=\sum v_{1 s} x v_{2 s} x v_{3 s}$ with each $v_{r s}$ in $A$. Another calculation then shows that

$$
\delta-\sum_{s} \delta_{s}=0,
$$

where $\delta_{8}=\left[v_{18}\right]^{22} \delta\left[v_{28}\right]^{12} \delta\left[v_{3 s}\right]^{11}$. Since $\delta_{s}$ is in the ideal $(\delta)^{2}$, we conclude from (3) that $\delta$ is $f$-regular. An application of Lemma 3 to (2) then shows that $\beta$ is $f$-regular, whereupon an application of Lemma 3 to (1) shows that $\alpha$ is $f$-regular. We have now proved that $A_{2}$ is $f$-regular.

The case in which $n$ is arbitrary can now be settled by an argument entirely analogous to that used at the corresponding point in the proof of Lemma 2 of [2]. The proof of Lemma 4 is therefore complete.

Now let $A$ be any ring. It follows from the preceding lemma that 
$(F(A))_{n}$ is an $f$-regular ideal of $A_{n}$ and therefore $(F(A))_{n} \subseteq F\left(A_{n}\right)$. On the other hand, the proof of the inclusion $F\left(A_{n}\right) \subseteq(F(A))_{n}$ may be carried through, with but minor (and obvious) modifications, as in the proof of the corresponding result (page 168) of [2]. We thus obtain the following

THEOREM 2. If $A_{n}$ is the complete matrix ring of order $n$ over the ring $A$, then $F\left(A_{n}\right)=(F(A))_{n}$.

3. The position of $F(A)$ in the ring. We proceed next to an investigation of the position of $F(A)$ in the ring $A$. The following theorem shows that, at the present time at least, one is unable to describe with precision the location of $F(A)$ with respect to the Jacobson radical $J(A)$ of $A$ (see the Introduction).

THEOREM 3. The following statements are equivalent:

(i) $J(A) \cap F(A)=0$ for every ring $A$.

(ii) Every f-regular ring is semi-simple.

(iii) Every simple ring is semi-simple.

(iv) For every radical ring $A$ which is generated by a single nonzero element, $A^{2} \neq A$.

Proof. Clearly (i) implies (ii). Suppose then that (ii) holds and that $A$ is a simple ring. Then $(a)^{2}=(a)$ for every $a \in A$ so that $A$ is $f$-regular and therefore semi-simple. Thus (ii) implies (iii). Now assume (iii) and let $A$ be a radical ring which is generated by the nonzero element $a$. By Zorn's lemma there is an ideal $M$ of $A$ which is maximal in the family of ideals of $A$ which exclude $a$. Then $M$ is a maximal ideal so that $A / M$ is a radical ring whose only ideals are itself and zero. Statement (iii) then requires that $(A / M)^{2}=0$, which means that $A^{2} \neq A$. Hence (iii) implies (iv). Suppose finally that there exists a ring $A$ such that $J(A) \cap F(A)$ contains a nonzero element $a$ and consider the ideal $B=(a)$ of $A$. Since $a \in F(A),(a)=(a)^{3}$ $\subseteq(a)_{B}$ as well as $(a)_{B} \subseteq(a)$; hence $B=(a)_{B}$. Moreover, $B$ is a radical ring since $B \subseteq J(A)$. But $B^{2}=B$ so that $B$ is a ring which contradicts (iv) and we conclude that (iv) implies (i). The proof is now complete.

We consider next the relationship between $F=F(A)$ and the prime radical $N=N(A)$ of McCoy [5]. If $B=N(A) \cap F(A)$, then $N(B)=B$ by $[5$, Theorem 4$]$ and $B$ is an $f$-regular ring by the remark following Theorem 1 above. But a ring is $f$-regular if and only if each of its ideals is an intersection of prime ideals [1, p. 147]. In particular then, $0=N(B)=B$ and therefore $N(A) \cap F(A)=0$. of $A$.

- We recall that $N(A)$ may be characterized as the intersection of all prime ideals 
If $B$ is an ideal of $A$, we define the annihilator of $B$ in $A$ to be the ideal $B^{*}$ consisting of all elements $a \in A$ such that $a B=B a=0$. It is clear that $F \cap F^{*}=0$. Moreover, since $N \cap F=0$ we have $N \subseteq F^{*}$ and $F \subseteq N^{*}$. Then $F\left(N^{*}\right)=F$ by Theorem 1 and $N\left(F^{*}\right)=N$ by the analogous result (Theorem 4 ) of [5].

We summarize these remarks in the following

Theorem 4. Let $F$ be the largest f-regular ideal of the ring $A$ and let $N$ be the prime radical of $A$. Then $N \cap F=0, F \cap F^{*}=0, F \subseteq N^{*}$, and $N \subseteq F^{*}$. Moreover, $F\left(N^{*}\right)=F$ and $N\left(F^{*}\right)=N$.

4. Rings with decending chain condition. We conclude this note by determining the effect on $F(A)$ of the descending chain condition for right ideals. We shall prove the following theorem.

THEOREM 5. If $A$ is a ring which satisfies the descending chain condition for right ideals, then $F(A)$ coincides with the largest regular ideal $M$ of $A$.

Proof. In the presence of this chain condition we may express $A$ as the (direct) sum

$$
A=M+M^{*} \text {. }
$$

Moreover, the ring $M^{*}$ is bound to its radical ${ }^{5}$ and the latter coincides with the radical $J=J(A)$ of $A$ (see Theorems 5 and 7 of [2]). Observe also that the chain condition requires that $J(A)=N(A)$ since in this case both $J(A)$ and $N(A)$ coincide with the classical radical. Therefore $J \cap F=0$ by Theorem 4 above. Thus $F \cap M^{*}$ is contained in the annihilator of $J$ in $M^{*}$, and this implies that $F \cap M^{*} \subseteq J$ since $M^{*}$ is bound to its radical. It follows that $F \cap M^{*}=0$. This fact, together with (4) and the obvious inclusion $M \subseteq F$, yields $F=M$.

\section{BIBLIOGRAPHY}

1. R. L. Blair, Ideal lattices and the structure of rings, Trans. Amer. Math. Soc. vol. 75 (1953) pp. 136-153.

2. B. Brown and N. H. McCoy, The maximal regular ideal of a ring, Proc. Amer. Math. Soc. vol. 1 (1950) pp. 165-171.

3. - Some theorems on groups with applications to ring theory, Trans. Amer. Math. Soc. vol. 69 (1950) pp. 302-311.

4. M. Hall, The position of the radical in an algebra, Trans. Amer. Math. Soc. vol. 48 (1940) pp. 391-404.

5. N. H. McCoy, Prime ideals in general rings, Amer. J. Math. vol. 71 (1949) pp. 823-833.

6. J. von Neumann, On regular rings, Proc. Nat. Acad. Sci. U.S.A. vol. 22 (1936) pp. 707-713.

University of California, Davis

- A ring $A$ is bound to its radical $J$ (in the sense of M. Hall [4]) in case $J^{*} \subseteq J$. 